\title{
A Note on Forecasting Daily Peruvian Stock Market Volatility Risk Using Intraday Returns
}

Mauricio Zevallos ${ }^{\mathrm{a}}$

a Department of Statistics, University of Campinas, Brazil

$\bowtie$ amadeus@unicamp.br

\begin{abstract}
In this paper I present a model to forecast the daily Value at Risk (VaR) of the Peruvian stock market (measured through the general index of the Lima Stock Exchange: the IGBVL) based on intraday (high-frequency) data. Daily volatility is estimated using realised volatility and I adopted a regression quantile approach to calculate one-step predicted VaR values. The results suggest that the realised volatility is a useful measure to explain the Peruvian stock market volatility and I obtained sound results using quantile regression for risk estimation.
\end{abstract}

Article History: Received: February 52019 / Revised: April 302019 / Accepted: July 152019

Keywords: High frequency data; Quantile Regression; Value-at-Risk.

JEL Classification: C22, C51, C53, C58

\section{Acknowledgements}

The author thanks the Editor-In-Chief and an anonymous referee for their many valuable comments. The author acknowledges financial support from the São Paulo Research Foundation (FAPESP) grant 2018/04654-9 and the support of the Centre for Applied Research on Econometrics, Finance and Statistics (CAREFS). A preliminary version of this work was presented at a seminar at the Central Reserve Bank of Peru in October 26, 2012; the author thanks the comments of the participants of this seminar. The author also thanks P. Baldoni for the assistance in preparing this manuscript. 


\section{Introduction}

Volatility has a vital role in risk management of financial assets such as stocks, indexes, currencies, etc. The volatility reflects the market risk structure and is a key element in pricing and portfolio allocation. Even though it is an unobservable variable, volatility can be estimated. Thus, since the 1980's, many models have been proposed to estimate daily volatility based on daily data, following the approaches of Engle (1982) using ARCH-type models, and Taylor (1986) through stochastic variance models. In the first approach, volatility is a function of past volatilities and past returns, whereas in the second volatility is a latent variable.

With the availability of records of intraday price movements, the so called tick by tick data or high frequency data, new approaches have emerged to estimate daily volatility. Taylor and Xu (1997) and Andersen and Bollerslev (1998) proposed estimating daily volatility using the realised volatility, measured as the sum of the squared intraday returns. The main difference between this approach and proposals based on daily data is the character of the realised volatility as observable. As such, it can be modelled and predicted.

The modelling and use of realised volatility have been intensively studied. Some topics of investigation are: modifications of the original measure of realised volatility (as the sum of squared intraday returns) in order to account for market microstructure effects (bid-ask bounces, price discreteness or non-synchronous trading); the study of statistical properties of realised volatility; modelling and forecasting realised volatility; and estimation of the Value at Risk (VaR) and daily return density forecast using realised volatility. Useful reviews of these subjects can be found in McAleer and Medeiros (2008) and Liu et al. (2015).

Academic literature about the use of high frequency data to model daily volatility and risk estimation of the Peruvian stock market is very scarce and, as far as I know, the only published work about this topic is Rodríguez $(2017 \mathrm{a})^{1}$. The purpose of the paper is to fill this gap and extend previous studies in which the Peruvian stock market risk has been estimated through the VaR using just daily data; see for example Zevallos (2008), del Carpio and Zevallos (2010), and Rodríguez (2017b).

Specifically, this paper uses realised volatility for risk management, calculating the Value at Risk by means of a quantile regression method proposed by Hua and Manzan (2013). This method was chosen because its versatility to handle non-Gaussian data, breaks and outliers. For this purpose, I study the general index of the Lima Stock Exchange, IGBVL, which is a value-weighted index (based on value 100 on December 30, 1991) and is commonly considered the benchmark of the Peruvian stock market. The results suggest that the realised volatility is a useful measure to explain the Peruvian stock market volatility, and I obtained sound backtesting results using quantile regression for risk estimation.

A different path is followed by Rodríguez (2017a), where models with random levels shifts and genuine long memory, proposed by Varneskov and Perron (2018), are applied to model daily volatility and to predict Value at Risk on the Peruvian stock market.

The remainder of this paper is organised as follows. Section 2 describes the method to calculate

\footnotetext{
${ }^{1}$ Unpublished references are Zevallos (2012) and Téllez et al. (2015).
} 
the realised volatility. Section 3 presents the estimation of one-step ahead daily Value at Risk prediction. These methods are applied in Section 4 on intraday IGBVL returns. Finally, Section 5 concludes.

\section{Realised Volatility}

Taylor and Xu (1997) and Andersen and Bollerslev (1998), among others, advocated that daily volatility may be estimated using intraday returns. Thus, each trading day $(t=1, \ldots, T)$ is divided into $\Delta$-minute intervals. Let $P_{t, i}$ be the price at the end of the $i$-th interval of day $t$ for $i=1, \ldots, M$ and let $P_{t, 0}$ be the first price of day $t$. The (continuously compounded) intraday returns in percentage, $r_{t, i}$, are calculated as:

$$
r_{t, i}=100\left[\ln \left(P_{t, i}\right)-\ln \left(P_{t, i-1}\right)\right], \quad i=1, \ldots, M,
$$

and $r_{t, 0}=100\left[\ln \left(P_{t, 0}\right)-\ln \left(P_{t-1, M}\right)\right]$. Thus, the total number of intraday returns ${ }^{2}$ is $N=(M+1) T$.

The realised variance or realised volatility on day $t, v_{t}$, is defined as:

$$
v_{t}^{2}=\sum_{i=0}^{M} r_{t, i}^{2}
$$

Assume that log-prices follow a continuous stochastic volatility model: $d Y_{s}=\left(\mu+\beta \sigma_{t}\right) d s+$ $\sigma_{s} d W_{s}$ where $\mu$ is the drift, $\beta$ is the risk premium, $\sigma_{s}^{2}$ is the instantaneous variance at time $s$ and $W_{s}$ is a standard Wiener process. Under certain conditions, Andersen and Bollerslev (1998) demonstrated that the realised variance $v_{t}^{2}$ is a consistent estimator of $\sigma_{t}^{2}=\int_{(t-1) \delta}^{t \delta} \sigma_{s}^{2} d s$. Therefore, the realised volatility estimates the total amount of variation in the time interval $\delta$ (here one day).

In practice, to calculate the realised volatility one has to specify the sampling frequency $\Delta$. As discussed by Giot and Laurent (2004) and Hardle et al. (2008), to name a few, using a very high sampling frequency (e.g., 30 seconds, 1 minute, etc.) may introduce bias in the variance estimate due to the so called market microstructure effects: bid-ask bounces, price discreteness or non-synchronous trading. On the other hand, sampling at small frequencies (e.g., 10, 15 or 30 minutes) may produce small bias but at the cost of high variability in the variance estimator. As a compromise between these two types of sampling, Andersen et al. (2003) and Liu et al. (2015) advocated the use of 5-minute returns to estimate daily realised volatility.

Statistical properties of the realised volatility and daily returns standardised by the square root of the realised volatility have been studied by several authors; see for example Hardle et al. (2008). Daily returns in percentage $\left(R_{t}\right)$ are defined using the closing prices as follows:

$$
R_{t}=100\left[\ln \left(P_{t, M}\right)-\ln \left(P_{t-1, M}\right)\right], \quad t=1, \ldots, T .
$$

Then the (daily) standardised returns are,

$$
z_{t}=\frac{R_{t}}{v_{t}}, \quad t=1, \ldots, T
$$

\footnotetext{
${ }^{2}$ Returns out of the official trading hours, called overnight returns, are usually discarded.
} 
A very well known model for daily returns is the product model, defined as:

$$
R_{t}=\sigma_{t} \epsilon_{t}
$$

where $\left\{\epsilon_{t}\right\}$ is an independent identically distributed sequence (IID) with zero mean and unit variance. Therefore, if realised volatility captures all the dependence in the data, one expects the estimated standardised returns $\left(\hat{z}_{t}\right)$

$$
\hat{z}_{t}=\frac{R_{t}}{\hat{v}_{t}}, \quad t=1, \ldots, T
$$

to be independent.

\section{Value at Risk Forecasting}

The Value at Risk (VaR) measures the potential loss in value of a risky asset or portfolio over a defined period for a given confidence interval. For instance, if the VaR of an asset is $\$ 10$ million in one-day, with $95 \%$ confidence level, there is only a $5 \%$ chance that the value of the asset will drop more than $\$ 10$ million during any given day.

Under the realised volatility paradigm, realised volatility estimates are considered observed values. As a consequence, time series models can be fitted to these values. To estimate the VaR at time $t+1$, one approach consists of predicting first the realised volatility at $t+1, \hat{v}_{t+1}^{2}$, based on the information until time $t$. Then one calculates the $\operatorname{VaR} \alpha \%$ as the (1- $\alpha)$-quantile of the distribution of the standardised returns. Typical models for predicting realised volatility are the ARFIMA model, proposed by Hosking (1981) and Granger and Joyeux (1980), and the HAR model proposed by Corsi (2009).

An interesting alternative to the two-step procedure above is the quantile regression approach. Assume that daily returns $R_{t}$ follow a location-scale model $R_{t}=\mu+\sigma_{t} \epsilon_{t}$ where $\mu$ is a constant, $\sigma_{t}$ is the conditional standard deviation on day $t$ and $\left\{\epsilon_{t}\right\}$ is an IID sequence with zero mean and variance one. Then, the $\tau$-quantile conditional on the information at time $t$ satisfies:

$$
Q_{t}(\tau)=\mu+\sigma_{t} Q_{\epsilon}(\tau)
$$

where $Q_{\epsilon}(\tau)$ is the $\tau$-quantile of the distribution of the disturbances $\epsilon_{t}$. Hua and Manzan (2013) assume that the conditional mean and the quantiles of the error are constants. Therefore, the return quantile varies over time only through changes in the conditional standard deviation of the process. In addition, they proposed to explain $\sigma_{t}$ by a set of observable variables at time $t$, including the realised volatility, and to estimate the model via quantile regression. In this paper, because the small size of the dataset, I adopt the following simple specification:

$$
Q_{t+1}(\tau)=\alpha_{0}(\tau)+\alpha_{1}(\tau) R_{t}+\alpha_{2}(\tau) v_{t}
$$

where $v_{t}$ is the square root of the realised volatility defined in (2). Thus, $Q_{t+1}$ depends on both the previous daily return and realised volatility. Once the parameters of the quantile regression model (7) - (8) are estimated, the VaR at time $t+1$ with $(1-\tau)$ confidence level is given by $\hat{Q}_{t+1}(\tau)$. 


\section{Empirical Application}

In this section I apply the proposed methods using high frequency Peruvian stock market data.

Intraday index figures of the IGBVL were collected from the Bloomberg database for the period from 15 September, 2011 to 22 August, 2012. This period is interesting because exhibits different volatility levels. To calculate realised volatilities, in this paper I consider $\Delta=15 \mathrm{~min}$, so for each day I have 36 intraday returns. The election of this sampled frequency was motivated by the small quantity of available intraday data. For instance, the reported indexes were very infrequent, reproducing a very well known characteristic of the Peruvian stock market: low liquidity.

Once the realised volatilities and daily returns were calculated as described in Section 2, performing $T=238$, I estimated the standardised returns, $\hat{z}_{t}$, by (6). Although not shown, the autocorrelation functions of $\hat{z}_{t}$ and $\hat{z}_{t}^{2}$ do not reveal serial correlation. Moreover, applying the Ljung-Box test with 10 lags on $\hat{z}_{t}$ and $\hat{z}_{t}^{2}$ led to $p$-values of 0.84 and 0.93 , respectively (for 20 lags the $p$-values are 0.88 and 0.50 , respectively). Therefore, it seems that the realised volatility captures the dependence structure of daily returns.

In order to obtain one-step ahead $95 \%$ VaR forecasts, I estimated model (8) using the quantreg package ( $\mathrm{R}$ Core Team, 2017). The estimates when $\tau=0.05$ using 15-min realised volatilities and daily returns are given in Table 1 . Here it can be seen that the estimates of the return and volatility are highly significant and that the effects are as expected. For instance, small returns and high realised volatilities imply low VaRs.

Table 1

Estimated quantile regression model.

\begin{tabular}{lrcrc}
\hline Coefficient & Estimate & Std. Error & $t$-value & $p$-value \\
\hline$\alpha_{0}(0.05)$ & -0.526 & 0.427 & -1.232 & 0.22 \\
$\alpha_{1}(0.05)$ & 0.474 & 0.234 & 2.025 & 0.04 \\
$\alpha_{2}(0.05)$ & -2.252 & 0.976 & -2.308 & 0.02 \\
\hline
\end{tabular}

Figure 1 presents the one-step predicted 95\% VaRs for daily IGBLV returns via quantile regression (red line). As can be seen, the predicted VaRs mimic very well the variability of the returns. In addition, the percentage of violations (times that the return is below the VaR) is $5.46 \%$, so the estimated model presents good results in terms of backtesting. As a comparison, I also estimated the VaRs using a popular method employed by practitioners: the RiskMetrics ${ }^{T M}$, see Longerstaey and More $(1995)^{3}$. The percentage of violations using RiskMetrics is $7.14 \%$, much bigger than the nominal value of $5 \%$, and as can be seen in Figure 1, the RiskMetrics VaRs are not adaptive enough in face of small returns which occurs after (very) large returns. For instance, once a large return appears, the next VaRs are often unnecessarily large when small returns occur. In contrast, the VaRs calculated by the proposed method in this paper

\footnotetext{
${ }^{3}$ Calculations were performed using the R function RMfit.R described in Tsay (2013), assuming a conditional Gaussian distribution.
} 


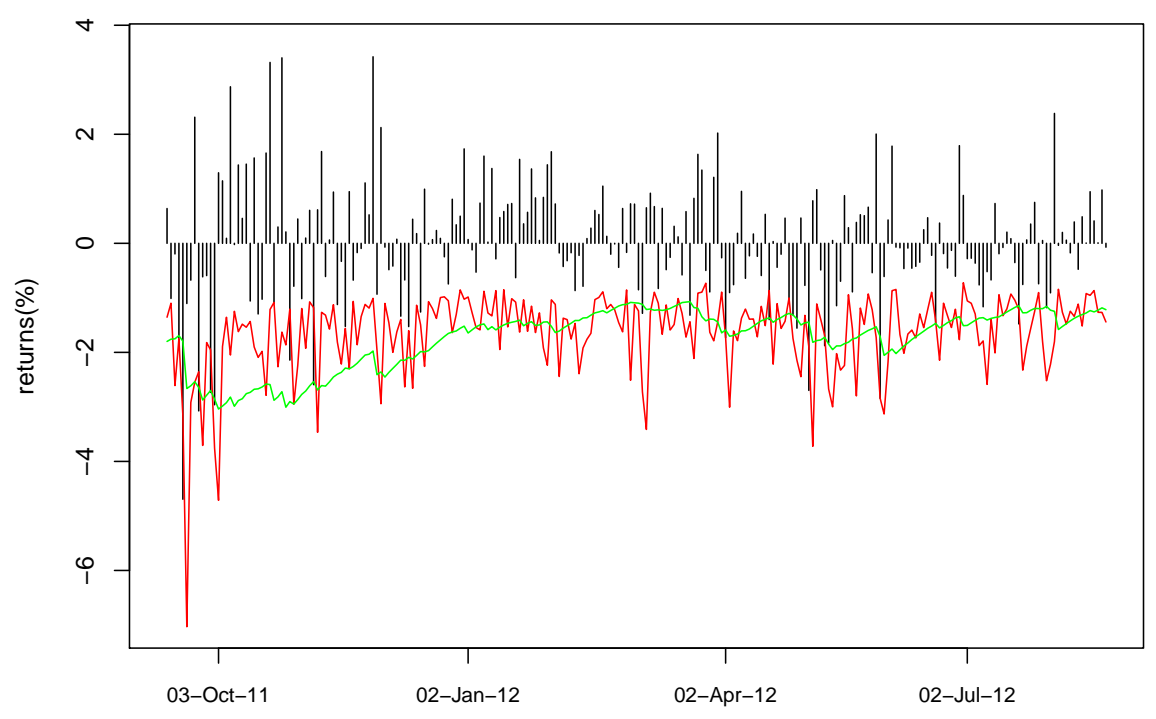

Figure 1. One-step ahead daily VaR prediction. Daily IGBVL returns in vertical lines, quantile regression VaRs in red and RiskMetrics VaRs in green.

are adaptive to the size of returns and are bigger than the RiskMetrics VaRs in periods of high volatility. Therefore, for this sample, in periods of great market uncertainty the quantile method is prefered, while in periods of medium or small uncertainty the RiskMetrics is a sound alternative. However, overall the quantile method is preferred given the small percentage of violations.

\section{Conclusions}

In this paper I applied quantile regression methods which use realised volatilities to forecast the Peruvian stock market risk. The main findings can be summarised as follows: the realised volatility is a useful measure to explain the market volatility of the IGBVL, and sound results are obtained by using a quantile regression approach for VaR estimation.

The results were obtained using a small sample (approximately one year of daily returns), so studies involving larger samples would be welcome. Other issues for further research are to study the convenience of using different realised volatility estimators and intraday frequencies; see Liu et al. (2015). This is especially important given the liquidity characteristics of the assets in the Peruvian stock market. Another issue for further research is to compare the Value at Risk estimates given by the proposed method in the paper and the method applied by Rodríguez (2017a). 


\section{References}

Andersen, T., and Bollerslev, T. (1998). Answering the skeptics: Yes, standard volatility models do provide accurate forecasts. International Economic Review 39(4), 885-905.

Andersen, T., Bollerslev, T., Diebold, F., and Labys, P. (2003). Modeling and forecasting realized volatility. Econometrica 71(2), 579-626.

Corsi, F. (2009). A simple approximate long-memory model of realized volatility. Journal of Financial Econometrics 7(2), 174-196.

del Carpio, C., and Zevallos, M. (2010) Estimación de Capital por Riesgo de Precio: Evaluando metodologías para el caso peruano (in Spanish) Revista Estudios Económicos 19, Central Reserve Bank of Peru, 42-56.

Engle, R. (1982). Autoregressive Conditional Heteroscedasticity with Estimates of the Variance of the United Kingdom Inflation. Econometrica 50(4), 987-1007.

Giot, P., and Laurent, S. (2004). Modelling daily Value-at-Risk using realized volatility and ARCH type models. Journal of Empirical Finance 11(3), 379-398.

Granger, C., and Joyeux, R. (1980). An introduction to long-memory time series models and fractional differencing. Journal of Time Series Analysis 1(1), 15-29.

Hardle, W., Hautsch, N., and Pigorsch, U. (2008). Measuring and Modeling Risk Using HighFrequency Data. SFB Discussion Paper 2008-045, Humboldt-Universitat zu Berlin.

Hosking, J. (1981). Fractional differencing. Biometrika 68(1), 165-76.

Hua, J., and Manzan, S. (2013). Forecasting the Return Distribution Using High-Frequency Volatility Measures. Journal of Banking 83 Finance 37(11), 4381-4403.

Liu, L., Patton, A., and Sheppard, K. (2015). Does anything beat 5-minute RV? A comparison of realized measures across multiple asset classes. Journal of Econometrics 187(1), 293-311.

Longerstaey, J., and More, L. (1995). Introduction to RiskMetrics ${ }^{T M}$. Fourth edition. New York: Morgan Guaranty Trust Company.

McAleer, M., and Medeiros, M. (2008). Realised Volatility: A Review. Econometric Reviews 27(1-3), 10-45.

R Core Team (2017). R: A language and environment for statistical computing. R Foundation for Statistical Computing, Vienna, Austria. URL http://www.R-project.org/.

Rodríguez, G. (2017a). Modeling Latin-American stock and Forex markets volatility: Empirical application of a model with random level shifts and genuine long memory. The North American Journal of Economics and Finance 42, 393-420.

Rodríguez, G. (2017b). Extreme Value Theory: An Application to the Peruvian Stock Market Returns. Revista de Métodos Cuantitativos para la Economía y la Empresa 23, 48-74.

Taylor, S. (1986). Modelling Financial Time Series, John Wiley and Sons, Chichester.

Taylor, S. and Xu, X. (1997). The Incremental Volatility Information in One Million Foreign Exchange Quotations. Journal of Empirical Finance 4(4), 317-340.

Téllez, G., Najarro, R. and Rodriguez, G. (2015). Modelos de Duración y Valor en Riesgo usando datos Peruanos del Mercado de Valores de alta frecuencia. Communication presented at the XXXIII Encuentro de Economistas del Banco Central de Reserva del Perú, U. del

\section{F PUCP}


Pacífico, Lima, Peru.

Tsay, R. (2013). An Introduction to Analysis of Financial Data with R. John Wiley \& Sons, Hoboken, New Jersey.

Varneskov, R. and Perron, P. (2018). Combining long memory and level shifts in modelling and forecasting the volatility of asset returns. Quantitative Finance 18(3), 371-393.

Zevallos, M. (2008). Estimación del Riesgo Bursátil Peruano. Economia 31(61), 109-126.

Zevallos, M. (2012). Realised Volatility in the Peruvian Stock Market. Mimeo, University of Campinas, Brazil. 